\title{
ORIGINAL
}

\section{Challenges in assessing the burden of sepsis and understanding the inequalities of sepsis outcomes between National Health Systems: secular trends in sepsis and infection incidence and mortality in Germany}

C. Fleischmann-Struzek ${ }^{1 *} \mathbb{0}$, A. Mikolajetz² , D. Schwarzkopf ${ }^{1}$, J. Cohen ${ }^{3}$, C. S. Hartog ${ }^{4,5}$, M. Pletz ${ }^{6}$, P. Gastmeier 7,8 and K. Reinhart ${ }^{1,2,9}$

(C) 2018 The Author(s)

\begin{abstract}
Purpose: Sepsis contributes considerably to global morbidity and mortality, while reasons for its increasing incidence remain unclear. We assessed risk adjusted secular trends in sepsis and infection epidemiology in Germany.

Methods: Retrospective cohort study using nationwide German hospital discharge data. We assessed incidence, outcomes and trends of hospital-treated sepsis and infections between 2010 and 2015. Sepsis was identified by explicit ICD-10 sepsis codes. As sensitivity analysis, results were compared with sepsis cases identified by implicit sepsis coding (combined infection and organ dysfunction codes).
\end{abstract}

Results: Among 18664877 hospital admissions in 2015, 4213116 (22.6\%) patients had at least one infection code. There were 320198 patients that had explicit sepsis codes including 136542 patients with severe sepsis and septic shock; 183656 patients were coded as sepsis without organ dysfunction. For patients with explicitly coded sepsis (including severe sepsis), or with severe sepsis alone, mortality rates over the period 2010-2015 decreased from 26.6 to $23.5 \%$, and from 47.8 to $41.7 \%$, respectively.

Conclusions: Sepsis and infection remain significant causes of hospital admission and death in Germany. Sepsisrelated mortality is higher and has declined to a lesser degree than in other high-income countries. Although infection rates steadily increased, the observed annual increase of sepsis cases seems to result, to a considerable degree, from improved coding of sepsis.

Keywords: Sepsis, Septic shock, Epidemiology, Secular trends

\section{Introduction}

Sepsis is the common final pathway to death from most infections [1]. Every year, sepsis causes more than 6

\footnotetext{
*Correspondence: carolin.fleischmann@med.uni-jena.de

${ }^{1}$ Center for Sepsis Control and Care, Jena University Hospital, Am Klinikum 1, 07747 Jena, Germany

Full author information is available at the end of the article
}

million deaths worldwide [2] and it is among the most expensive conditions treated in the hospital [3, 4]. National reports from UK and Australia have identified sepsis as a major cause of avoidable deaths in the hospital $[5,6]$. The World Health Organization recognized sepsis as a major public health problem [7] and in a recent resolution urged all UN member states to improve sepsis prevention, recognition, and management [8]. It 
specifically demands improved epidemiological surveillance to better understand the true burden of sepsis. Currently, most studies on sepsis epidemiology rely on ICD-based administrative data and suggest a continuously rising incidence in the range of $5-10 \%$ annually $[4,9]$. These marked increases were questioned because they were not matched by comparable increases in ICDdocumented infection rates [10]. Assessing the incidence of sepsis is challenging and may be dependent on the framework used [11]. In 2016, new sepsis definitions ("sepsis-3") were proposed by a task force of the Society of Critical Care Medicine and the European Society of Intensive Care Medicine [1]. According to sepsis-3, a life-threatening organ dysfunction caused by a dysregulated host response to infection is now termed "sepsis", which corresponds to "severe sepsis" according to the traditional definition. Sepsis without organ dysfunction and SIRS criteria (systematic inflammatory response syndrome) no longer form part of the new definitions. These changes are likely to impact estimates in sepsis epidemiology and may also require updated strategies to identify sepsis cases in administrative data [2]. Recent studies, which estimated sepsis incidence by sepsis-3 criteria using electronic health records (EHR) and chart review, found incidence rates in the US (approximately 517/100 000 population) [12] and Sweden (780/100 000 population) [13] that were at least 3.5-fold higher than current estimates for the global [2] and national incidence of severe sepsis based on administrative data in Germany [4]. These studies found that less than $50 \%$ of sepsis patients with organ dysfunction in the US and only $15 \%$ in Sweden received an explicit ICD-code for sepsis [12, 13]. Therefore, the combination of infection and organ dysfunction codes [14], the implicit case identification method, may be less prone to coding bias than the currently used explicit sepsis codes [4]. Therefore, we aimed (1) to assess national trends in infection and sepsis incidence and mortality rates in Germany between 2010 and 2015 based on explicit sepsis codes, and (2) to compare the results with estimates based on ICD-coded infection and organ dysfunction (implicit method) as sensitivity analysis.

\section{Methods}

\section{Data source}

DRG statistics is a nationwide all-payer database including complete inpatient data from nearly all acute-care hospitals in Germany. Military or prison hospitals are excluded. It is accessible via remote data processing by the German Federal Statistical Office. Each hospitalization is treated as an individual entry and contains one principal ICD-10 German Modification (ICD-10-GM) diagnosis, up to 89 secondary ICD-10-GM diagnoses, up to 100 OPS codes (classification of operations and procedures), length of hospital stay, type of admission and discharge, and patient demographics.

\section{Description of patients}

Cases with unknown age or gender were excluded from analysis. We identified patients of all ages between 2010 and 2015 using all available primary and secondary discharge ICD-10 codes according to the following groups (Fig. 1): (1) all infection codes, (2) explicit sepsis codes including severe sepsis and septic shock [4], (3) explicit severe sepsis codes including septic shock, and (4) explicit sepsis without organ dysfunction. Infection codes were modified from Angus [14] and comprised $>1200$ ICD-10-GM codes (Supplement 1) including explicit sepsis codes. Infections were classified as respiratory, abdominal, wound and soft tissue, genitourinary, central nervous, device-related, pregnancy-related, cardiovascular, and non-specific infections. Explicit sepsis codes comprised R-codes and microbiological codes for sepsis, Supplement 2. Explicit severe sepsis including septic shock was identified by codes R65.1 and R57.2. All explicitly coded sepsis cases with no code for severe sepsis and septic shock were labeled as explicitly coded sepsis without organ dysfunction. In Germany, R-codes are defined according to modified 1992 sepsis criteria [15]. To avoid overcoding, the use of R65.0 is restricted to cases with positive blood culture and 2/4 SIRS criteria or to cases with 4/4 SIRS criteria in case of negative blood cultures [16]. As sensitivity analysis, we compared results for implicit severe sepsis coding (combining codes for infection and organ dysfunction [14]). The implicit case identification method includes explicit sepsis codes. Organ dysfunctions were assessed by 27 ICD-10-GM codes (Supplement 3). We assessed patient demographics; site of infection; underlying comorbidity using the Charlson Comorbidity Index (CCI) [17]; resource use including ICU care, mechanical ventilation, renal replacement therapy, surgical treatment, palliative care treatment (for all OPS codes, see Supplement 4), hospital length of stay; discharge disposition (for the definition of discharge categories, see Supplement 5); and hospital mortality.

\section{Statistical analyses}

Data were analyzed using SAS ${ }^{\circledR}$ (Version 9.4; SAS Institute, Cary, NC, USA) and R (Version 3.4.0; R Core Team, Wien, Austria) and are presented as percentages, numbers, medians and interquartile ranges (IQRs), or means and standard deviations (SDs). We calculated annual population-based incidences that were directly standardized to the German population structure as of 31 December 2010 on the basis of nationwide population data of the Federal Statistical Office for 2010-2015. Given the 


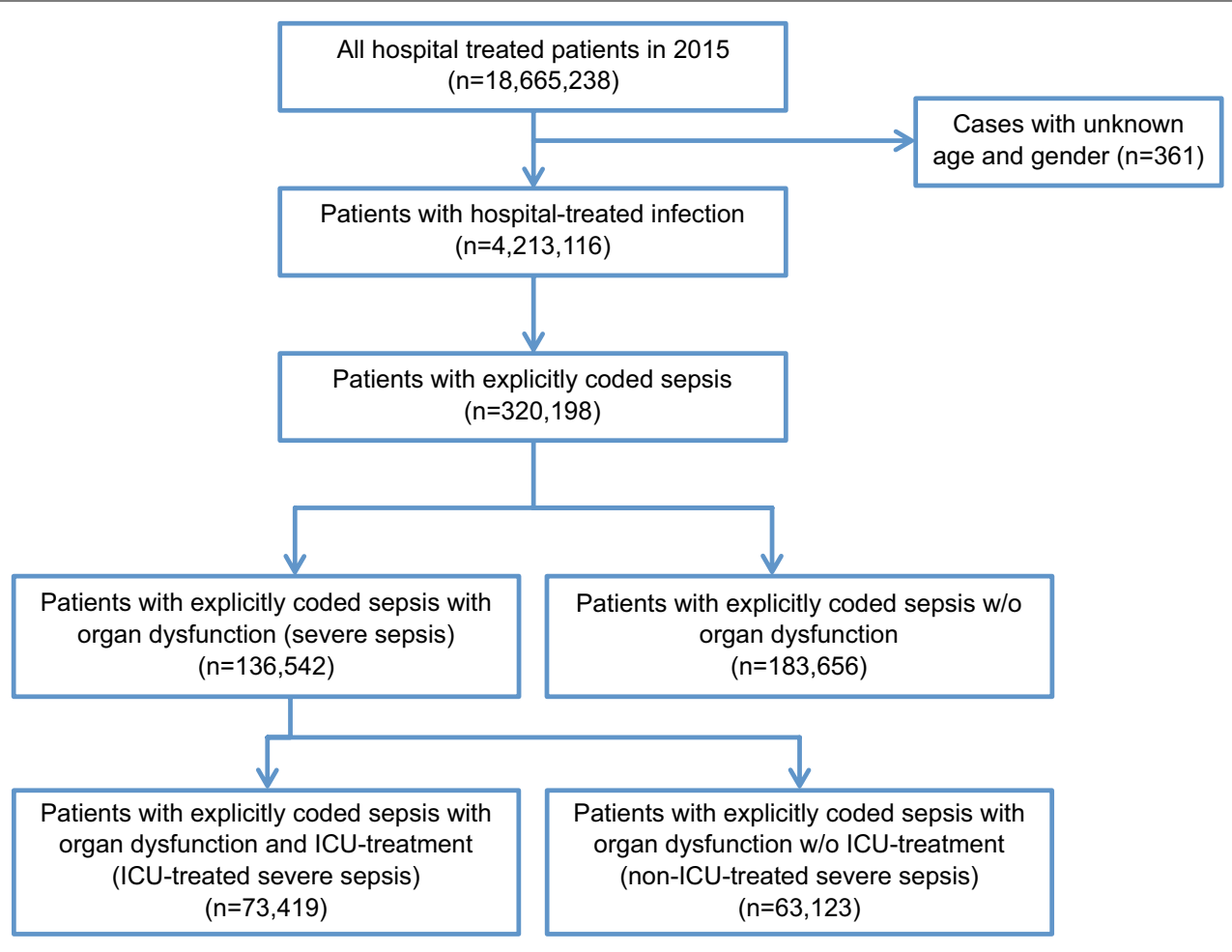

Fig. 1 Flow of case identification and absolute number of cases in 2015

large size of the data set, we did not perform inferential statistics, as all comparisons were likely to be statistically significant. Since changes in mortality might be related to changes in case-mix, we calculated risk-adjusted mortality rates for patients with explicitly coded sepsis. For this purpose a risk-model previously described [18] was fitted. Based on probability of mortality predicted by this model, we calculated risk-standardized mortality rates for each year.

\section{Results}

\section{Infection and explicitly coded sepsis}

Among all 18664877 hospital admissions in a population of 82175684 in Germany in 2015, 4213116 (22.6\%) of all patients had at least one infection code (Fig. 1). Respiratory infections were the most common infections, followed by genitourinary and abdominal infections (Supplementary Fig. 7A and B). There were 320198 patients that had explicitly coded sepsis. Of the 136542 patients with severe sepsis and septic shock, $53.8 \%$ were treated in the ICU. Also, 183656 patients were coded as sepsis without organ dysfunction. Infections and explicitly coded sepsis were present in $60.1 \%$ and $17.7 \%$ of all hospitalizations that culminated in death, respectively (Supplementary Fig. 9). Demographics and characteristics of patients with sepsis and infection are shown in
Table 1 and Supplementary Tables 6.1-6.8. Hospital admission rates of patients with infection or explicitly coded sepsis increased continuously between 2010 and 2015 (Supplementary Fig. 8). Population-based incidence rates of hospital-treated infection increased by a mean of $1.8 \%$ annually from 4515 to 4923 per 100000 population (Fig. 2). Among patients with infection, the largest annual increase was found in respiratory and device-related infections (3.7\% and 3.8\%), respectively. Population-level incidence rates for patients with explicitly coded sepsis and explicitly coded severe sepsis increased by an annual mean of $5.7 \%$ from 280 to 370 per 100000 population and by $7.9 \%$ from 108 to 158 per 100000 population, respectively. Mean patient age and the proportion of patients with multiple comorbidities $(\mathrm{CCI}>1)$ increased in all categories (Supplementary Tables 6.1-6.8). The proportion of surgical patients among infection, explicitly coded sepsis and explicitly coded severe sepsis patients declined over time. Median hospital length of stay also declined in these groups (from 8 to 7 days, 14 to 12 days and 17 to 15 days, respectively). The proportion of patients requiring mechanical ventilation or renal replacement therapy also declined in patients with explicitly coded sepsis and explicitly coded severe sepsis, but increased slightly in patients with infection (Table 1). Mortality of patients with infection did not change substantially (6.0-6.1\%). 
Table 1 Demographics of patients with explicitly coded sepsis (including severe sepsis), explicitly coded severe sepsis, explicitly coded sepsis without organ dysfunction, treated in German hospitals in 2010 and 2015

\begin{tabular}{|c|c|c|c|c|c|c|c|c|}
\hline & \multicolumn{2}{|l|}{ Infection } & \multicolumn{2}{|c|}{$\begin{array}{l}\text { Explicitly coded sep- } \\
\text { sis (including severe } \\
\text { sepsis) }\end{array}$} & \multicolumn{2}{|c|}{$\begin{array}{l}\text { Explicitly coded } \\
\text { severe sepsis (ICU } \\
\text { and non-ICU treat- } \\
\text { ment) }\end{array}$} & \multicolumn{2}{|c|}{$\begin{array}{l}\text { Explicitly coded } \\
\text { sepsis without organ } \\
\text { dysfunction }\end{array}$} \\
\hline & 2010 & 2015 & 2010 & 2015 & 2010 & 2015 & 2010 & 2015 \\
\hline$n$ & $3,691,241$ & $4,213,116$ & 229,214 & 320,198 & 87,973 & 136,542 & 141,241 & 183,656 \\
\hline Deaths & 221098 & 255573 & 61068 & 75227 & 42084 & 56875 & 18984 & 18352 \\
\hline Incidence per 100000 (age-/sex-standardized) & 4515 & 4923 & 280 & 370 & 108 & 158 & 173 & 212 \\
\hline Deaths per 100000 (age-/sex-standardized) & 270 & 288 & 75 & 86 & 51 & 65 & 23 & 21 \\
\hline Age in years, mean (SD) & $58.8(26.5)$ & $60.9(25.6)$ & $64.8(22.3)$ & $67.3(20.2)$ & $68.5(16.6)$ & $70.0(15.8)$ & $62.6(25.0)$ & $65.3(22.8)$ \\
\hline Female gender, $\%$ & 52.9 & 52.0 & 44.5 & 43.2 & 42.1 & 41.3 & 46.0 & 44.6 \\
\hline $\mathrm{CCl}$, median (IQR) & $1(0 ; 3)$ & $1(0 ; 3)$ & $2(1 ; 4)$ & $2(1 ; 4)$ & $3(1 ; 4)$ & $3(1 ; 5)$ & $2(1 ; 4)$ & $2(1 ; 4)$ \\
\hline \multicolumn{9}{|l|}{ Comorbidities, $\%$} \\
\hline None & 41.1 & 38.0 & 20.7 & 18.0 & 14.7 & 12.6 & 24.4 & 22.0 \\
\hline 1 & 22.2 & 22.4 & 24.6 & 24.3 & 23.8 & 22.6 & 25.2 & 25.4 \\
\hline $2-4$ & 34.1 & 36.5 & 49.9 & 52.3 & 55.1 & 57.6 & 46.6 & 48.4 \\
\hline$>4$ & 2.6 & 3.1 & 4.8 & 5.5 & 6.3 & 7.2 & 3.9 & 4.2 \\
\hline Surgical treatment, \% & 31.6 & 29.6 & 38.2 & 33.8 & 50.8 & 43.1 & 30.4 & 26.9 \\
\hline Hospital LOS, days, median (IQR) & $8(4 ; 14)$ & $7(4 ; 13)$ & $14(8 ; 27)$ & $12(7 ; 24)$ & $17(8 ; 33)$ & $15(7 ; 29)$ & $13(7 ; 24)$ & $11(7 ; 20)$ \\
\hline ICU admission, \% & 8.5 & 9.3 & 33.4 & 32.7 & 56.4 & 53.8 & 19.1 & 17.0 \\
\hline RRT, \% & 1.9 & 2.0 & 11.4 & 9.9 & 22.0 & 18.7 & 4.8 & 3.3 \\
\hline Mechanical ventilation, $\%$ & 4.9 & 5.8 & 25.3 & 24.0 & 45.2 & 42.2 & 12.9 & 10.6 \\
\hline Palliative care, \% & 0.5 & 1.0 & 0.9 & 1.8 & 0.7 & 1.7 & 1.1 & 2.0 \\
\hline Mortality, \% & 6.0 & 6.1 & 26.6 & 23.5 & 47.8 & 41.7 & 13.4 & 10.0 \\
\hline \multicolumn{9}{|l|}{ Discharge disposition of survivors, $\%$} \\
\hline Regular* & 86.5 & 85.1 & 74.0 & 73.9 & 61.3 & 63.1 & 78.7 & 79.1 \\
\hline Other hospital & 4.6 & 5.1 & 12.8 & 12.8 & 20.3 & 20.1 & 9.9 & 9.3 \\
\hline Hospice & 0.1 & 0.1 & 0.2 & 0.3 & 0.2 & 0.3 & 0.2 & 0.3 \\
\hline Rehab & 3.0 & 2.5 & 6.4 & 4.8 & 11.2 & 7.7 & 4.5 & 3.4 \\
\hline Nursing home & 3.8 & 4.8 & 5.1 & 6.6 & 5.5 & 7.4 & 5.0 & 6.2 \\
\hline Other & 2.1 & 2.3 & 1.5 & 1.7 & 1.5 & 1.5 & 1.5 & 1.7 \\
\hline
\end{tabular}

IQR interquartile range, $C C I$ charlson comorbidity index, LOS length of stay, ICU intensive care unit, $R R T$ renal replacement therapy

* Regular discharge includes regular termination of treatment with discharge at home, with or without post-discharge treatment intended

For patients with explicitly coded severe sepsis (including septic shock), mortality decreased from 47.8 to $41.7 \%$ (Fig. 3). Likewise, mortality decreased from 49.1 to $45.2 \%$ for patients with explicit severe sepsis codes who received ICU treatment. Mortality of patients with explicit severe sepsis codes treated outside the ICU decreased from 46.2 to $37.5 \%$. Risk-standardized mortality rates for explicitly coded sepsis, severe sepsis and sepsis without organ dysfunction are displayed in Supplementary Fig. 10. Both the crude and risk-standardized mortality showed a similar decrease over time, although the risk-standardized mortality decreased more in patients with explicitly coded sepsis compared to the crude mortality. Discharge dispositions for patients with explicitly coded severe sepsis remained virtually unchanged except for a decrease in the number of discharges to rehabilitation facilities from 11.2 to $7.7 \%$, which was accompanied by an increase of palliative care from 0.7 to $1.7 \%$.

\section{Sensitivity analysis: implicit severe sepsis coding}

In 2015, implicit severe sepsis coding identified 1166061 cases $(6.3 \%$ of all hospital admissions, compared to $0.7 \%$ for explicitly coded severe sepsis), of which $16.9 \%$ died. The mean annual increase in population-level incidence was $7.3 \%$ from 942 to 1336 per 100000 population. Compared to explicitly coded severe sepsis, patients identified by implicit severe sepsis coding had fewer comorbidities and surgical procedures, and required less mechanical ventilation, renal replacement therapy and ICU admission. A majority of implicitly identified severe sepsis 

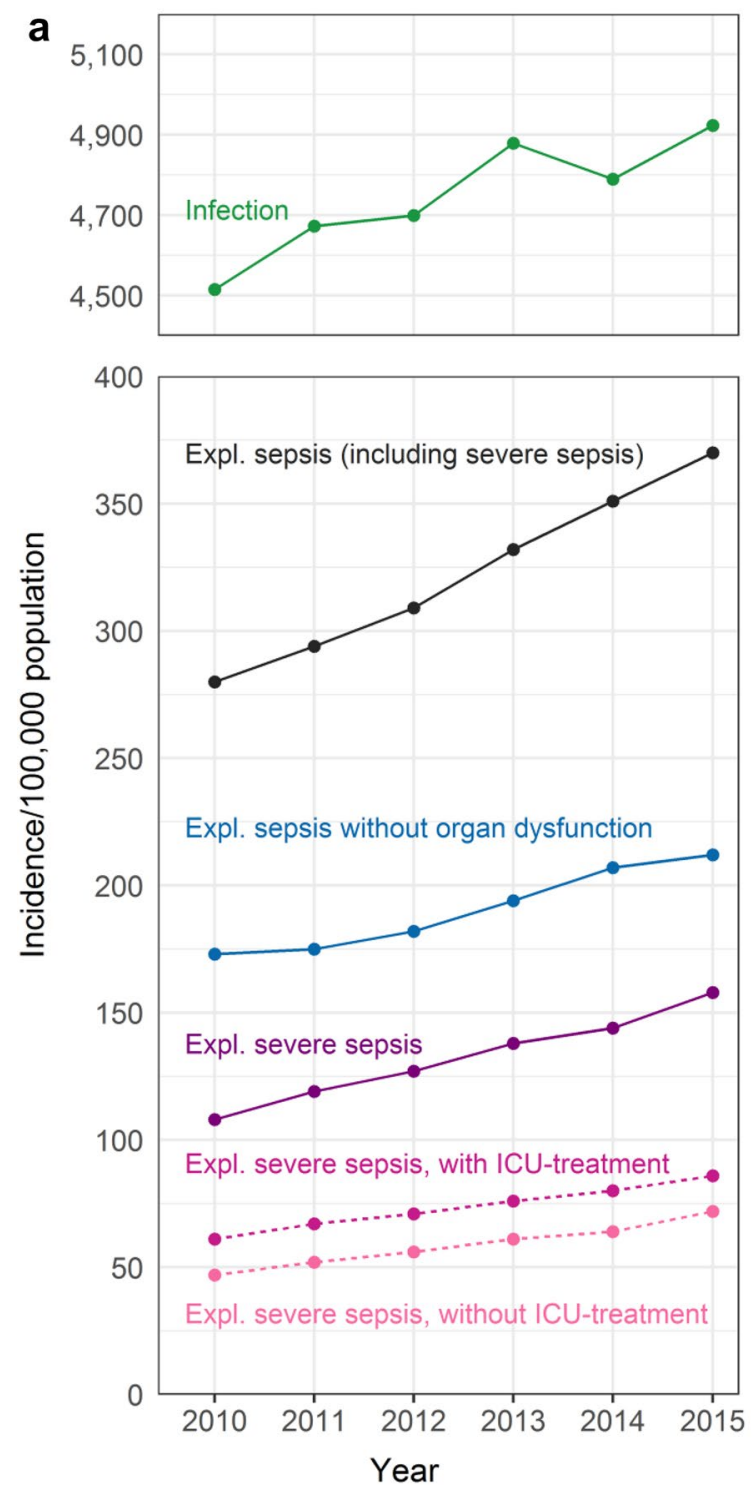

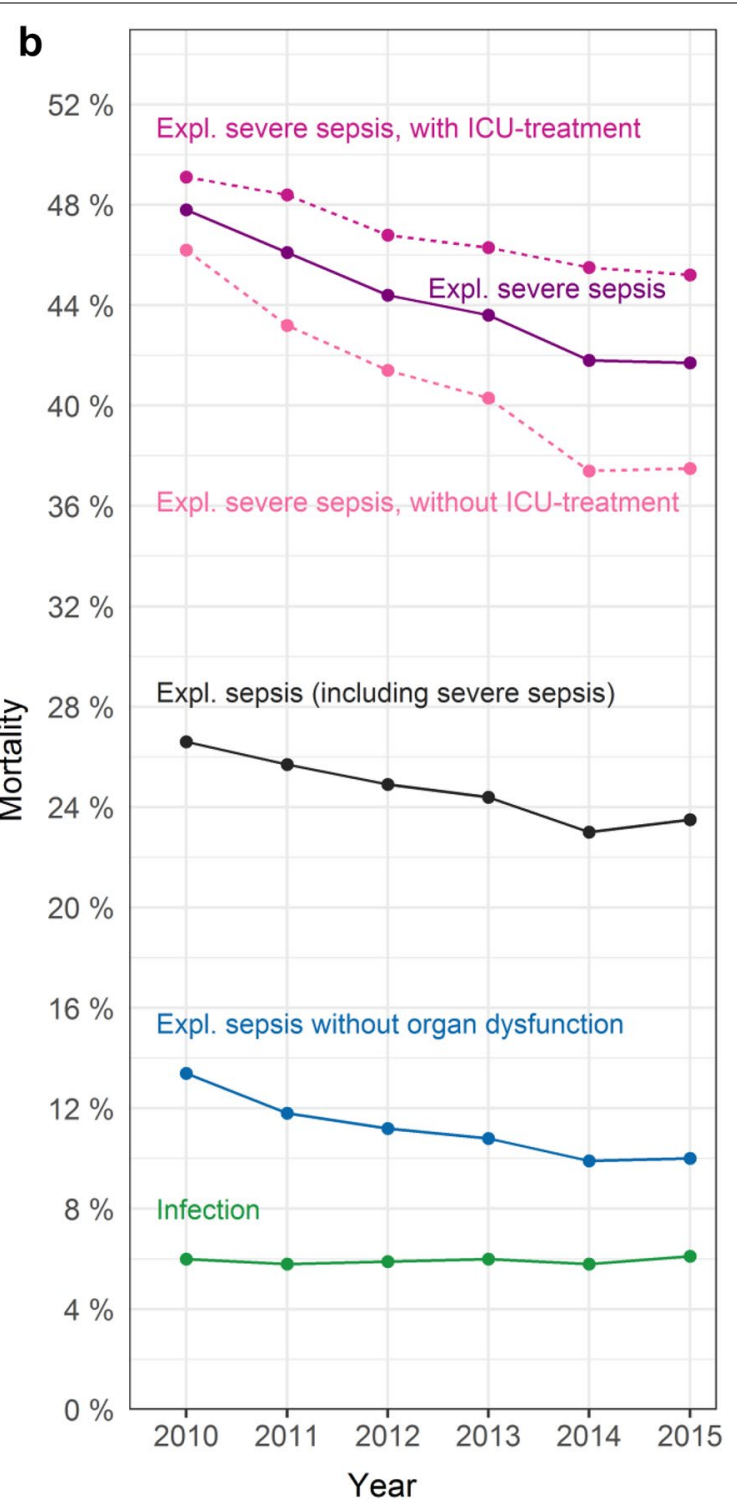

Fig. 2 Population-based incidence and in-hospital mortality rates in Germany between 2010 and 2015. Presented are patients with infection, explicitly coded sepsis (including severe sepsis and septic shock), explicitly coded sepsis without organ dysfunction, explicitly coded severe sepsis (including septic shock) and explicitly coded severe sepsis with ICU treatment and without ICU treatment patients did not receive an explicit sepsis code at hospital discharge (Fig. 3). These patients had lower mortality rates and fewer ICU admission rates.

\section{Discussion}

The main findings of this retrospective nation-wide analysis of German hospital discharge data are: (1) that the annual incidence of explicitly coded severe sepsis increased between 2010 and 2015 by $7.9 \%$, and is poorly matched with the observed annual increase in infection rate of only $1.8 \%$; (2) severe sepsis mortality decreased by $2.6 \%$ between 2010 and 2015, whereas discharge dispositions for patients with explicitly coded severe sepsis remained virtually unchanged; and (3) a sensitivity analysis on implicitly coded severe sepsis cases, which are less prone to coding bias, revealed significantly higher case rates, but similar secular trends with a mean annual increase in sepsis incidence of $7.3 \%$ between 2010 and 2015.

The estimated incidence of 158 explicitly coded sepsis cases per 100000 population in Germany in 2015 is 3-5 times lower than in other countries (Table 2). Rhee 


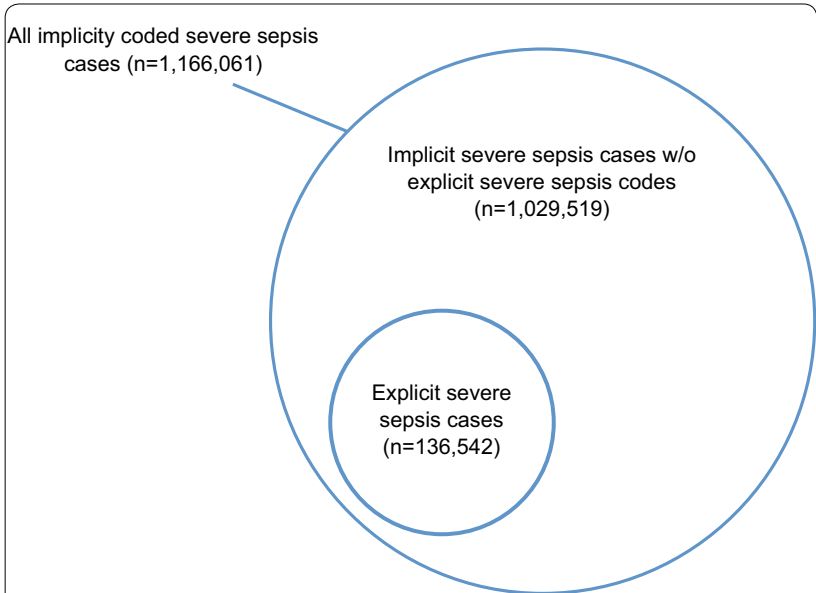

Fig. 3 In 2015, a majority of patients identified by implicit sepsis coding received no explicit severe sepsis code during their hospital stay

and colleagues found an estimated incidence of approximately 517/100 000 population in the US in 2014 based on clinical sepsis-3 criteria in EHR [12]. Similarly, chart review from a database including all patients treated with antibiotics in Sweden in 2015 yielded an estimated incidence for sepsis-3 cases of 780/100 000 and of 687/100 000 population for traditional severe sepsis [13]. Interestingly, the underestimation of cases by up to 3.5 -fold by explicit coding observed in a single center validation study in Germany is quite similar to that [19]. The underestimation resulted from missed clinical sepsis diagnoses, but was also related to the fact that to avoid upcoding, coding of sepsis in Germany is limited to cases with blood cultures drawn and requires the presence of four SIRS criteria in case of negative blood cultures. Arguably, this may prevent the coding of less severe sepsis cases in Germany, but may also miss a relevant proportion of sepsis cases, which are SIRS negative [20]. The underestimation of real sepsis rates by explicit coding of severe sepsis in Germany is supported by the finding in Sweden that $28 \%$ of infection patients (687 of $2425 / 100000$ population) based on chart review fulfilled clinical criteria for severe sepsis [13] and by Walkey et al. who in administrative data found a proportion of $15 \%$ (535 of 3480/100 000 population) [21]. By comparison, in the present study only $3.2 \%$ of patients with any infection code were coded as severe sepsis (158 of 4923/100 000). Moreover, undercoding may also explain that in Germany explicitly coded severe sepsis was present only in $13.4 \%$ of hospitalizations that culminated in death compared to $35 \%$ in the US [12].

The observed annual increase in explicitly coded severe sepsis cases in Germany is similar to the increase of explicitly coded severe sepsis cases in the US. In the
US, the validity of the reported increase in sepsis incidence and concurrent decrease in sepsis mortality based on administrative data was questioned and attributed to increased coding of less severe cases due to greater awareness and billing incentives [10]. To clarify this controversy Rhee and colleagues compared sepsis trends from 2009 to 2014 in EHR by clinical sepsis-3 criteria to the results from explicit and implicit coding strategies [12]. Surprisingly, they found sepsis hospital admission rates of $6 \%$ by clinical criteria in EHR and approximately $12 \%$ by implicit coding compared to only approximately $3 \%$ by explicit coding. Hospital admission rates based on clinical sepsis criteria remained stable over the 5-year observation period but increased by approximately $50 \%$ for both explicitly and implicitly coded sepsis [12]. Interestingly, our sensitivity analysis found similar trends for implicit severe sepsis identified by infection and organ dysfunction coding as for explicit severe sepsis. This suggests that an improved coding of sepsis may contribute to the observed annual increase of sepsis cases, but there may also be objective reasons for an increase in rates of severe infection and sepsis rates. Our study found an overall increase in hospital-treated infections and in particular in respiratory, genitourinary tract and device-related infections, which are known to be major sources of sepsis in German ICUs [22]. Reports from the US and England found rising hospital admission rates for patients with infections $[21,23]$. The increasing proportion of elderly patients with multiple comorbidities who are more susceptible to sepsis and have higher mortality rates may also contribute to an increase $[4,14]$.

Hospital mortality in Germany seems to be higher compared to other health economies. Compared to the US, the hospital mortality of patients with severe sepsis identified by explicit coding in Germany is higher and the decline over time is less (34-24\% [12] and 47.8-41.7\%, respectively). In addition, according to data from Australia and England that are derived from nationwide ICU registries, which are not prone to billing incentives and changes in coding practices, hospital mortality of severe sepsis between 2000 and 2012 decreased from 35.0 to $18.4 \%$ and from 45.5 to $32.1 \%$, respectively $[24,25]$. In comparison, hospital mortality of explicitly coded severe sepsis patients admitted to ICU in Germany between 2010 and 2015 decreased only from 49.1 to $45.2 \%$. Similar trends were found for risk-adjusted mortality rates of explicitly coded severe sepsis patients, suggesting that there was no major change in case-mix.

Several factors may account for these surprising findings such as differences in patient populations, availability of health care resources, the quality of the health care system especially in respect to the standards and 


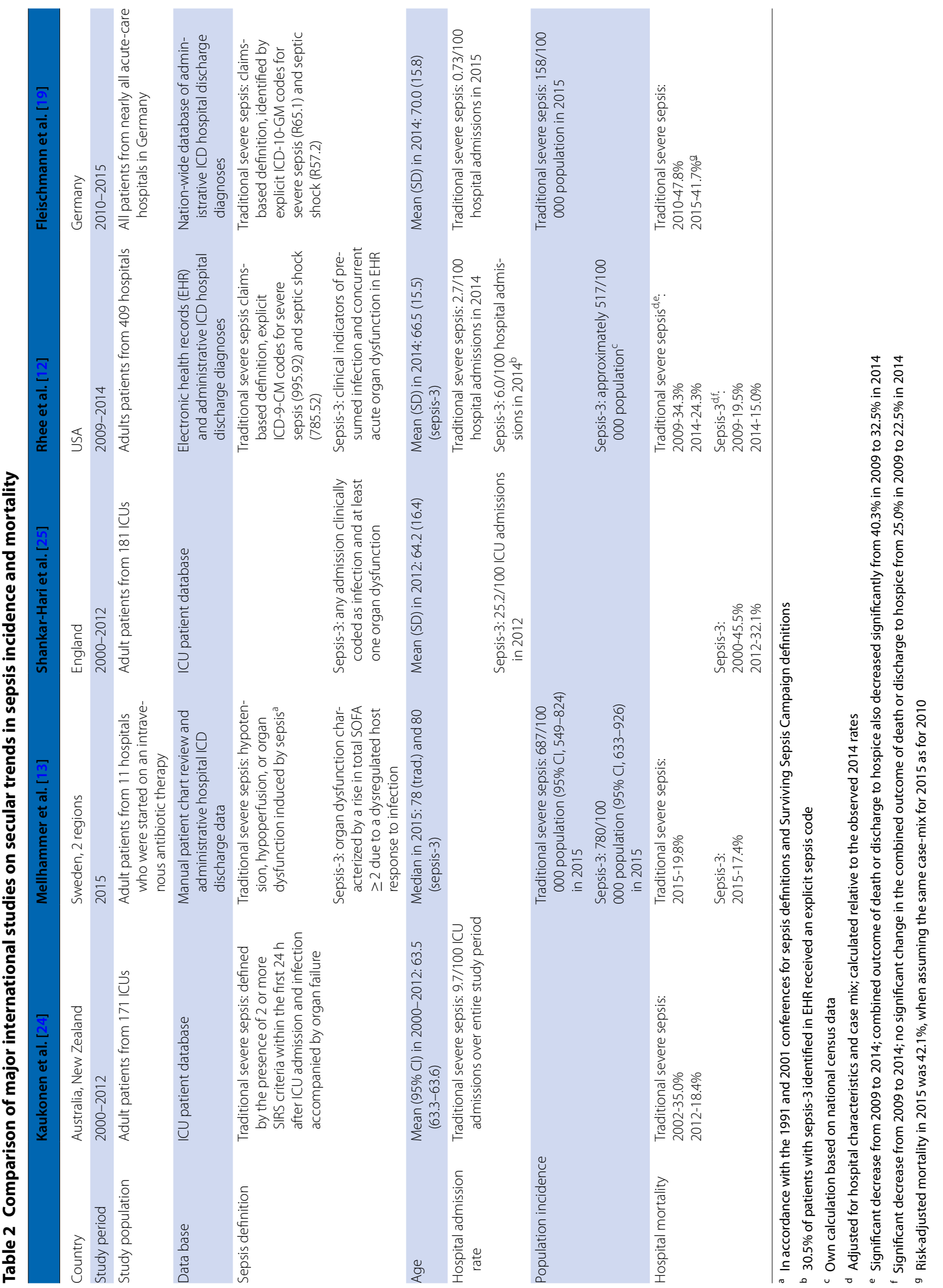


systematic efforts for infection control and prevention, and the management of deteriorating patients in hospitals.

The mean age of explicitly severe sepsis patients in the respective study populations of these four countries was highest in Germany (69.4 years) and lowest in Australia (63.5 years). However, the difference in sepsis mortality between these three countries is consistent over the whole range of age categories from $<40$ to $>80$ years (Supplementary Fig. 10 based on personal communications from Ch. Rhee [12], R. Bellomo [24], and M. Shankar-Hari [25]). Although a significant proportion of sepsis patients die after discharge from hospital and thus a shorter length of hospital stay may result in lower inhospital mortality rates, the mean difference in length of hospital stay between Germany and Australia and the US of between 1.5 and 5 days is unlikely to be a significant contributor to the observed differences.

The total number of hospital beds in Germany is between 2- and 4-times higher than in Australia, UK and the US, and Germany has the highest number of ICU beds closely followed by the US [26]. This may contribute to the higher hospital admission rates and longer length of hospital stay in Germany, but does not support the notion that in high-income countries the numbers of ICU and hospital beds might have an impact on sepsis outcomes. Some infections that may lead to sepsis such as pneumonia and influenza may be reduced by vaccination of elderly against pneumococci and influenza [27, 28]. Interestingly, the vaccination rate against influenza for elderly above age 65 years who belong to the at risk population is only $35.3 \%$ in Germany compared to $69.1,71.1$ and $74.6 \%$ in the US, UK and Australia, respectively [29]. Similar differences apply for the vaccination rates against pneumococci in the elderly (Germany 31.4\% [30], US 63.6\% [31], England 69.8\% [32] and Australia 56\% [33]). Furthermore, it has been estimated that 1500 to 4500 deaths through sepsis in Germany could be avoided each year by prevention of health care associated infections [34]. Quality improvement programs including mandatory performance measures [35] and the introduction of rapid response teams, which have become standard in the US, UK and Australia, have demonstrated a sustained improvement in sepsis outcomes [36-41]. Similar nationwide efforts and structures in Germany are missing.

In Germany, quality improvement and control is primarily left to the discretion of health care providers, department heads, and the self-governing bodies of the German health system. Likewise, the low number and shortage of infectious diseases specialists and clinical microbiologists in Germany, as well as the poor standards in microbiology diagnosis in terms of numbers of blood cultures per patient-days and time to results due to the large number of remote nonresident microbiological laboratories in Germany may contribute to the observed differences in sepsis outcomes [42, 43].

Given the assumption that explicit sepsis coding leads to a considerable underestimation of sepsis cases, a major limitation of our study is that it did not directly compare clinical sepsis criteria to administrative data. However, a validation study [19], a quality improvement study comprising $>4000$ severe sepsis patients of 40 German hospitals [44], and two representative epidemiologic studies $[22,45]$, that were all based on chart reviews, found high hospital mortality rates for severe sepsis patients in the range of over $40-50 \%$. This suggests that the mortality rate that we found by explicit sepsis coding is quite similar to that from chart reviews and that the observed differences in comparison to other similar health economies remain true independently from the methodological approach.

In summary, we find that infection and sepsis remain significant causes of hospital admission and death in Germany, whereas an improved coding of sepsis may contribute to the observed annual increase of sepsis cases of $5.7 \%$. The rate of decline of sepsis-related mortality appears less than in some comparable health economies. Finally, there remains a striking difference between the absolute mortality of sepsis in Germany and some other countries. The reasons for this are complex but may relate to delays in the widespread introduction of quality improvement programs and, hence, delays in early diagnosis and treatment, but also to poor adherence to quality standards in blood culture testing and a shortage of infectious disease specialists, rather than inadequacies in the provision of ICU and hospital beds.

\section{Electronic supplementary material}

The online version of this article (https://doi.org/10.1007/s00134-018-5377-4) contains supplementary material, which is available to authorized users.

\section{Author details \\ ${ }^{1}$ Center for Sepsis Control and Care, Jena University Hospital, Am Klinikum 1 07747 Jena, Germany. ${ }^{2}$ Department for Anesthesiology and Intensive Care Medicine, Jena University Hospital, Am Klinikum 1, 07740 Jena, Germany. \\ ${ }^{3}$ Brighton and Sussex Medical School, University of Sussex, Brighton BN1 9PX, UK. ${ }^{4}$ KLINIK BAVARIA Kreischa, An der Wolfsschlucht 1-2, 01731 Kreischa, Germany. ${ }^{5}$ Klinik für Anäesthesie mS operative Intensivmedizin, Charité Universitätsmedizin Berlin, Charitéplatz 1, 10117 Berlin, Germany. ${ }^{6}$ Institute of Infectious Diseases and Infection Control, Jena University Hospital, Am Klinikum 1, 07740 Jena, Germany. ${ }^{7}$ Institute of Hygiene and Environmental Medicine, Charité Berlin, Berlin, Germany. ${ }^{8}$ German National Reference Centre for the Surveillance of Nosocomial Infections, Berlin, Germany. ${ }^{9}$ BIH Visiting Professor/Stiftung Charité Klinik für Anäesthesie mS operative Intensivmedizin, Charité Universitätsmedizin Berlin, Charitéplatz 1, 10117 Berlin, Germany.}

\section{Acknowledgements}

Data source:"Research Data Centres of the Federal Statistical Office and the statistical offices of the Länder, DRG statistics 2007-2015". We thank Ch. Rhee, R. Bellomo and M. Shankar-Hari for providing additional data from their studies. 


\section{Compliance with ethical standards}

\section{Conflicts of interest}

$\mathrm{KR}$, DS and CF receive grants from the German Federal Ministry of Education and Research (BMBF) via the Center for Sepsis Control and Care (CSCC; FKZ: 01EO1002 and 01EO1502). AM receives grants from the German Federal Ministry of Education and Research (BMBF) via InfectControl2020 (FKZ: 03ZZ0819B). MP is partly supported by a grant of the Federal Ministry of Education and Research (BMBF) KliFo 2.0 (FKZ: 01 KI1501). KR is unpaid chairman of the Global Sepsis Alliance. KR is a shareholder of InflaRx Jena, a University spin-off that develops adjunctive therapies for systemic inflammation and an advisor to Adrenomed Henningsdorf/Berlin, Germany, who develop an antibody against Adrenomedullin to treat cardio-circulatory shock. The remaining authors have disclosed that they do not have any potential conflicts of interest. The funding sources had no involvement in the design, analysis, and writing of the present manuscript.

\section{Open Access}

This article is distributed under the terms of the Creative Commons Attribution-NonCommercial 4.0 International License (http://creativecommons.org/ licenses/by-nc/4.0/), which permits any noncommercial use, distribution, and reproduction in any medium, provided you give appropriate credit to the original author(s) and the source, provide a link to the Creative Commons license, and indicate if changes were made.

Received: 1 July 2018 Accepted: 15 September 2018 Published online: 4 October 2018

\section{References}

1. Singer M, Deutschman CS, Seymour CW, Shankar-Hari M, Annane D, Bauer M, Bellomo R, Bernard GR, Chiche JD, Coopersmith CM, Hotchkiss RS, Levy MM, Marshall JC, Martin GS, Opal SM, Rubenfeld GD, van der Poll T, Vincent JL, Angus DC (2016) The third international consensus definitions for sepsis and septic shock (Sepsis-3). J Am Med Assoc 315:801-810

2. Fleischmann C, Scherag A, Adhikari NK, Hartog CS, Tsaganos T, Schlattmann P, Angus DC, Reinhart K, International Forum of Acute Care T (2016) Assessment of global incidence and mortality of hospital-treated sepsis. Current estimates and limitations. Am J Respir Crit Care Med 193:259-272

3. Torio CM, Moore BJ (2016) National inpatient hospital costs: the most expensive conditions by payer, 2013: statistical brief \#204. Healthcare Cost and Utilization Project (HCUP) Statistical Briefs, Rockville

4. Fleischmann C, Thomas-Rueddel DO, Hartmann M, Hartog CS, Welte T, Heublein S, Heublein S, Dennler U, Reinhart K (2016) Hospital incidence and mortality rates of sepsis. Dtsch Arzteblatt Int 113:159-166

5. Burrell AR, McLaws ML, Fullick M, Sullivan RB, Sindhusake D (2016) SEPSIS KILLS: early intervention saves lives. Med J Aust 204(73):e71-e77

6. Goodwin A, Srivastava V, Shotton H, Protopapa K, Butt A, Mason M (2015) Just Say Sepsis! A review of the process of care received by patients with sepsis. NCEPOD (National Confidential Enquiry into Patient Outcome and Death), London

7. Reinhart K, Daniels R, Kissoon N, Machado FR, Schachter RD, Finfer S (2017) Recognizing sepsis as a global health priority —a WHO resolution. New Engl J Med 377:414-417

8. World Health Organisation Executive Board (EB140/12) (2017) Improving the prevention, diagnosis and clinical management of sepsis. http://apps. who.int/gb/ebwha/pdf_files/EB140/B140_12-en.pdf. Accessed 20 June 2017

9. Kumar G, Kumar N, Taneja A, Kaleekal T, Tarima S, McGinley E, Jimenez E, Mohan A, Khan RA, Whittle J, Jacobs E, Nanchal R (2011) Nationwide trends of severe sepsis in the 21st century (2000-2007). Chest 140:1223-1231

10. Rhee C, Gohil S, Klompas M (2014) Regulatory mandates for sepsis carereasons for caution. New Engl J Med 370:1673-1676

11. Seymour CW, Coopersmith CM, Deutschman CS, Gesten F, Klompas M, Levy M, Martin GS, Osborn TM, Rhee C, Warren DK, Watson RS, Angus DC (2016) Application of a framework to assess the usefulness of alternative sepsis criteria. Crit Care Med 44:e122-e130
12. Rhee C, Dantes R, Epstein L, Murphy DJ, Seymour CW, Iwashyna TJ, Kadri SS, Angus DC, Danner RL, Fiore AE, Jernigan JA, Martin GS, Septimus E, Warren DK, Karcz A, Chan C, Menchaca JT, Wang R, Gruber S, Klompas M, Program CDCPE (2017) Incidence and trends of sepsis in US hospitals using clinical vs claims data, 2009-2014. J Am Med Assoc 318:1241-1249

13. Mellhammar L, Wullt S, Lindberg A, Lanbeck P, Christensson B, Linder A (2016) Sepsis incidence: a population-based study. Open Forum Infect Dis 3. https://doi.org/10.1093/ofid/ofw207

14. Angus DC, Linde-Zwirble WT, Lidicker J, Clermont G, Carcillo J, Pinsky MR (2001) Epidemiology of severe sepsis in the United States: analysis of incidence, outcome, and associated costs of care. Crit Care Med 29:1303-1310

15. Reinhart $\mathrm{K}$, Brunkhorst FM, Bone HG, Bardutzky J, Dempfle CE, Forst $H$, Gastmeier P, Gerlach H, Grundling M, John S, Kern W, Kreymann G, Kruger W, Kujath P, Marggraf G, Martin J, Mayer K, Meier-Hellmann A, Oppert M, Putensen C, Quintel M, Ragaller M, Rossaint R, Seifert H, Spies C, Stuber F, Weiler N, Weimann A, Werdan K, Welte T (2010) Prevention, diagnosis, therapy and follow-up care of sepsis: 1st revision of S-2k quidelines of the German Sepsis Society (Deutsche Sepsis-Gesellschaft e.V. (DSG)) and the German Interdisciplinary Association of Intensive Care and Emergency Medicine (Deutsche Interdisziplinare Vereinigung fur Intensiv- und Notfallmedizin (DIVI)). Ger Med Sci. https://doi.org/10.3205/000103

16. Deutsches Institut für Medizinische Dokumentation und Information (2018) Was versteht man unter SIRS-Systemisches inflammatorisches response-syndrom? https://www.dimdi.de/dynamic/de/klassifikationen/ kodierfrage/Was-versteht-man-unter-SIRS-Systemisches-inflammatorisch es-Response-Syndrom-ICD-10-GMnbspNr.nbsp1007/. Accessed 09 Aug 2018

17. Charlson ME, Pompei P, Ales KL, MacKenzie CR (1987) A new method of classifying prognostic comorbidity in longitudinal studies: development and validation. J Chronic Dis 40:373-383

18. Schwarzkopf D, Fleischmann-Struzek C, Ruddel H, Reinhart K, ThomasRuddel DO (2018) A risk-model for hospital mortality among patients with severe sepsis or septic shock based on German national administrative claims data. PLoS One 13:e0194371

19. Fleischmann-Struzek C, Thomas-Ruddel DO, Schettler A, Schwarzkopf D, Stacke A, Seymour CW, Haas C, Dennler U, Reinhart K (2018) Comparing the validity of different ICD coding abstraction strategies for sepsis case identification in German claims data. PLoS One 13:e0198847

20. Kaukonen KM, Bailey M, Pilcher D, Cooper DJ, Bellomo R (2015) Systemic inflammatory response syndrome criteria in defining severe sepsis. New Engl J Med 372:1629-1638

21. Walkey AJ, Lagu T, Lindenauer PK (2015) Trends in sepsis and infection sources in the United States. A population-based study. Ann Am Thorac Soc 12:216-220

22. Engel $\mathrm{C}$, Brunkhorst FM, Bone HG, Brunkhorst $\mathrm{R}$, Gerlach $\mathrm{H}$, Grond $\mathrm{S}$, Gruendling M, Huhle G, Jaschinski U, John S, Mayer K, Oppert M, Olthoff D, Quintel M, Ragaller M, Rossaint R, Stuber F, Weiler N, Welte T, Bogatsch H, Hartog C, Loeffler M, Reinhart K (2007) Epidemiology of sepsis in Germany: results from a national prospective multicenter study. Intensive Care Med 33:606-618

23. Trotter CL, Stuart JM, George R, Miller E (2008) Increasing hospital admissions for pneumonia, England. Emerg Infect Dis 14:727-733

24. Kaukonen KM, Bailey M, Suzuki S, Pilcher D, Bellomo R (2014) Mortality related to severe sepsis and septic shock among critically ill patients in Australia and New Zealand, 2000-2012. J Am Med Assoc 311:1308-1316

25. Shankar-Hari M, Harrison DA, Rowan KM (2016) Differences in impact of definitional elements on mortality precludes international comparisons of sepsis epidemiology - a cohort study illustrating the need for standardized reporting. Crit Care Med 44(12):2223-2230

26. Wunsch H, Angus DC, Harrison DA, Collange O, Fowler R, Hoste EA, de Keizer NF, Kersten A, Linde-Zwirble WT, Sandiumenge A, Rowan KM (2008) Variation in critical care services across North America and Western Europe. Crit Care Med 36(2787-2793):e2781-e2789

27. Bonten MJ, Huijts SM, Bolkenbaas M, Webber C, Patterson S, Gault S, van Werkhoven $C H$, van Deursen AM, Sanders EA, Verheij TJ, Patton M, McDonough A, Moradoghli-Haftvani A, Smith H, Mellelieu T, Pride MW, Crowther G, Schmoele-Thoma B, Scott DA, Jansen KU, Lobatto R, Oosterman B, Visser N, Caspers E, Smorenburg A, Emini EA, Gruber WC, Grobbee DE (2015) Polysaccharide conjugate vaccine against pneumococcal pneumonia in adults. New Engl J Med 372:1114-1125 
28. Christenson B, Lundbergh P, Hedlund J, Ortqvist A (2001) Effects of a large-scale intervention with influenza and 23-valent pneumococcal vaccines in adults aged 65 years or older: a prospective study. Lancet 357:1008-1011

29. Organisation for Economic Co-operation and Development (2018) Influenza vaccination rates. https://data.oecd.org/healthcare/influenza-vacci nation-rates.htm. Accessed 19 Mar 2018

30. Poethko-Müller C, Schmitz R (2013) Impfstatus von Erwachsenen in Deutschland. Ergebnisse der Studie zur Gesundheit Erwachsener in Deutschland (DEGS1). Bundesgesundheitsblatt 56:845-857

31. Williams WW, Lu PJ, O'Halloran A, Kim DK, Grohskopf LA, Pilishvili T, Skoff TH, Nelson NP, Harpaz R, Markowitz LE, Rodriguez-Lainz A, Fiebelkorn AP (2017) Surveillance of vaccination coverage among adult populationsUnited States, 2015. MMWR Surveill Summ 66:1-28

32. Public Health England (2015) Pneumococcal polysaccharide vaccine (PPV) coverage report, England, April 2014 to March 2015. https://asset s.publishing.service.gov.uk/government/uploads/system/uploads/attac hment data/file/448406/hpr2615 ppv.pdf. Accessed 05 June 2018

33. Dyda A, Karki S, Hayen A, MacIntyre CR, Menzies R, Banks E, Kaldor JM, Liu B (2016) Influenza and pneumococcal vaccination in Australian adults: a systematic review of coverage and factors associated with uptake. BMC Infect Dis 16:515

34. Gastmeier P, Brunkhorst F, Schrappe M, Kern W, Geffers C (2010) Wie viele nosokomiale Infektionen sind vermeidbar? Dtsch Med Wochenschr 135(3):91-93

35. Sama A (2014) Quality sepsis care: ACEP's mission, advocacy and research in action. http://www.thecentralline.com/?p=3091. Accessed 20 Aug 2018

36. Seymour CW, Gesten F, Prescott HC, Friedrich ME, Iwashyna TJ, Phillips GS, Lemeshow S, Osborn T, Terry KM, Levy MM (2017) Time to treatment and mortality during mandated emergency care for sepsis. New Engl J Med 376:2235-2244
37. Liu VX, Fielding-Singh V, Greene JD, Baker JM, Iwashyna TJ, Bhattacharya J, Escobar GJ (2017) The timing of early antibiotics and hospital mortality in sepsis. Am J Respir Crit Care Med 196:856-863

38. Burrell AR, McLaws ML, Fullick M, Sullivan RB, Sindhusake D (2016) SEPSIS KILLS: early intervention saves lives. Med J Aust 204:73

39. Damiani E, Donati A, Serafini G, Rinaldi L, Adrario E, Pelaia P, Busani S, Girardis M (2015) Effect of performance improvement programs on compliance with sepsis bundles and mortality: a systematic review and meta-analysis of observational studies. PLoS One 10:e0125827

40. Jones DA, DeVita MA, Bellomo R (2011) Rapid-response teams. New Engl J Med 365:139-146

41. Evans IVR, Phillips GS, Alpern ER, Angus DC, Friedrich ME, Kissoon N, Lemeshow S, Levy MM, Parker MM, Terry KM, Watson RS, Weiss SL, Zimmerman J, Seymour CW (2018) Association between the New York sepsis care mandate and in-hospital mortality for pediatric sepsis. J Am Med Assoc 320:358-367

42. Kern W, Fätkenheuer G, Tacconelli E, Ullmann A (2015) Infectious diseases as a clinical specialty in Germany and Europe. Z Evid Fortbild Qual Gesundhwes 109(7):493-499

43. Fatkenheuer G, Kern WV, Salzberger B (2016) An urgent call for infectious diseases specialists. Infection 44:269-270

44. Bloos F, Ruddel H, Thomas-Ruddel D, Schwarzkopf D, Pausch C, Harbarth S, Schreiber T, Grundling M, Marshall J, Simon P, Levy MM, Weiss M, Weyland A, Gerlach H, Schurholz T, Engel C, Matthaus-Kramer C, Scheer C, Bach F, Riessen R, Poidinger B, Dey K, Weiler N, Meier-Hellmann A, Haberle HH, Wobker G, Kaisers UX, Reinhart K (2017) Effect of a multifaceted educational intervention for anti-infectious measures on sepsis mortality: a cluster randomized trial. Intensive Care Med 43(11):1602-1612

45. SepNet Critical Care Trials G (2016) Incidence of severe sepsis and septic shock in German intensive care units: the prospective, multicentre INSEP study. Intensive Care Med 42:1980-1989 